\title{
Bremsstrahlung Contribution in Compton Scattering from Heavy Metals*
}

\author{
Usha Mittal, B. K. Sharma, and R. K. Kothari \\ Department of Physics, University of Rajasthan, Jaipur (India) \\ B. L. Ahuja \\ Department of Physics, M. Regional Engineering College, Jaipur (India) \\ Z. Naturforsch. 48a, 348-351 (1993); received December 31, 1991
}

\begin{abstract}
Theoretical estimates of the spectral distribution and total intensity of the bremsstrahlung (BS) relative to the Compton yield have been determined for photoelectrons liberated in scattering experiments on heavy metals with $60 \mathrm{keV}$ radiation. Whereas the total-intensity calculations are based on the approach of Alexandropoulos et al., the spectral distribution has been computed using the Born approximation along with the Elwert and form factor corrections. These results are compared with another calculation based on the approach of Lee et al. Typical results for $\mathrm{Mo}, \mathrm{Ta}$, and $\mathrm{W}$ are presented. It is found that the relative contribution of BS from a W target is not trivially small, although $\mathrm{K}$-shell photo-ionisation is not possible at these energies.
\end{abstract}

Key words: Compton profile; Bremsstrahlung; Born approximation; Photoelectrons; Screened potential.

\section{Introduction}

There has been a revival of interest in the use of the Compton scattering technique for investigating the electronic structure of materials [1,2]. The Compton line shape, $J\left(p_{z}\right)$, is experimentally determined in such studies and can be computed theoretically in a direct manner by the double integration of the electron density in momentum space. The experimental determination of $J\left(p_{z}\right)$, however, is not straightforward. As has been discussed by several authors [1,2], the raw spectrum of the radiation scattered by the target has to be corrected for several factors, the most tedious and difficult of these being the corrections for the energy response of the spectrometer and multiple scattering in the sample. During the last decade or so, considerable progress has been made in handling these corrections, and raw data with a precision of a few tenths of a percent have been reported [3].

For heavy metals, it has been realised for over three decades that bremsstrahlung (BS) can have a considerable influence on Compton scattering data [4]. A few

* Presented at the Sagamore X Conference on Charge, Spin and Momentum Densities, Konstanz, Fed. Rep. of Germany, September 1-7, 1991.

Reprint requests to Prof. Dr. B. K. Sharma, Department of Physics, University of Rajasthan, Jaipur-302004 (India). years ago, Alexandropoulos et al. [5] re-examined the effects of bremsstrahlung in the inelastic photon scattering experiment. These authors calculated not only the total BS intensity $\left(I_{\mathrm{BS}}\right)$ but also the spectral distribution using Kramer's theory and have been able to reproduce the own measurements on $\mathrm{W}$ up to $30 \mathrm{keV}$ closely. These authors further concluded that the BS contribution relative to the Compton yield could exceed even $10 \%$, if one used $60 \mathrm{keV}$ gamma radiation with targets having an atomic number larger than 40 . Recently we have undertaken a systematic study of some $5 \mathrm{~d}$ metals using $60 \mathrm{keV}$ radiation [6,7]. It was therefore thought worthwhile to examine the effects of BS carefully for the accurate determination of $J\left(p_{z}\right)$ in these high- $Z$ metals. Accordingly, we have calculated the spectral distribution of BS using the first Born approximation and then applied the necessary corrections due to the Elwert factor and the form factor (screening). We have also computed this spectrum using the approach of Lee et al. [8], and the two results have been compared. The estimates of the BS contribution in the region of the Compton profile have been determined from these spectra for a number of metals of the $4 \mathrm{~d}$ and $5 \mathrm{~d}$ series.

In Sect. II we describe the method of calculation. In Sect. III our results and their discussion are presented. The conclusions are given in Section IV. 


\section{Method of Calculation}

The formulae for calculating the intensity and spectral distribution of BS by electrons are readily available [9], and hence we state here only the key relations used in this work. The non-relativistic differential BS cross-section for the emission of a photon with energy $w$ can be written as

$\frac{\mathrm{d} \phi_{w}}{\mathrm{~d} w}=\phi \cdot \frac{8}{3} \frac{1}{T_{0}} \frac{\mu}{w} \cdot \log \left[\frac{\left(\sqrt{T_{0}}+\sqrt{T_{0}-w}\right)^{2}}{w}\right]$,

where $\phi=Z^{2} r_{0}^{2} / 137.04, r_{0}=e^{2} /\left(m_{0} c^{2}\right), \mu=m_{0} c^{2}$, and $T_{0}$ is the kinetic energy of the primary electron. As pointed out in [9], this formula is valid only when $Z e^{2} / \hbar v \ll 1$ with $v$ being the velocity of the electron. Otherwise (1) must be multiplied by a factor (Elwert factor) given as

$f\left(\xi, \xi_{0}\right)=\frac{\xi}{\xi_{0}} \cdot \frac{1-e^{-2 \pi \xi_{0}}}{1-e^{-2 \pi \xi}} \approx \frac{\xi}{\xi_{0}}$,

where $\xi$ and $\xi_{0}$ respectively are $Z e^{2} / \hbar v$ and $Z e^{2} / \hbar v_{0}$.

Further, a correction due to screening of nuclear charge may become necessary [9] for low-energy photons and high- $Z$ materials (such as those involved here). The unscreened differential cross-section formula (1) may be corrected for screening effects by including the multiplicative factor $|F(q)|^{2}, F(q)$ being the form factor defined as [10]

$$
F(\boldsymbol{q})=1-\frac{1}{Z} \int \varrho(\boldsymbol{r}) e^{i \boldsymbol{q} \cdot \boldsymbol{r}} \mathrm{d} \boldsymbol{r},
$$

where $\int \varrho(\boldsymbol{r}) \mathrm{d} \boldsymbol{r}=Z, \boldsymbol{q}=\boldsymbol{p}_{1}-\boldsymbol{p}_{2}-\boldsymbol{k}$ and $\varrho(\boldsymbol{r})$ is the charge density of the electron cloud.

The total BS cross-section is then defined as

$$
\phi_{w}=\frac{1}{E_{0}} \int_{0}^{T_{0}} w \frac{\mathrm{d} \phi_{w}}{\mathrm{~d} w} \mathrm{~d} w,
$$

where $E_{0}=T_{0}+m_{0} c^{2}$.

In the present case we cannot make use of these relations directly, as electrons are liberated inside the sample owing to both photoelectric and Compton effects. Thus we have first to know the energies and (relative) yields of electrons generated via these two processes. This can, however, be determined from the cross-section for the photoionisation of different shells, Compton cross-section and incident photon flux. The knowledge of the photon flux is not necessary if we are only interested in relative intensities of BS and Comp- ton scattered radiation, because then the photon flux cancels. Further, at this low energy $(60 \mathrm{keV})$, the contribution of Compton electrons is almost negligible [5], as also discussed later. We thus consider only the BS from photoelectrons to determine $I_{\mathrm{BS}} / I_{\mathrm{C}}$. In order to obtain that ratio, we have used the following well known relations [11]:

$$
I_{\mathrm{BS}}^{\text {total }}=\sum_{i} N\left(w_{0}\right) \cdot \sigma_{\mathrm{pe}, i}\left(w_{0}, Z\right) \cdot k \cdot Z \cdot\left(w_{0}-E_{\mathrm{b}, i}\right)^{2}
$$

and

$$
I_{\mathrm{C}}^{\text {total }}=N\left(w_{0}\right) \cdot \sigma_{\mathrm{C}}\left(w_{0}, Z\right) \cdot w_{\mathrm{C}},
$$

where $I_{\mathrm{BS}}^{\text {total }}$ is the total intensity of BS owing to photoelectrons, $I_{\mathrm{C}}^{\text {total }}$ the total intensity of Compton radiation, $N\left(w_{0}\right)$ the incident flux of photons with energy $w_{0}$ and $\sigma_{\mathrm{pe}, i}$ the photoelectric cross-section for a particular shell $i$ with binding energy $E_{\mathrm{b}, i}$ of the target element with atomic number $Z$ for the incident-photon energy $w_{0}$. The constant $k \approx 10^{-3} \mathrm{MeV}^{-1}, \sigma_{\mathrm{C}}$ is the Compton cross-section and $w_{\mathrm{C}}$ the (average) energy of the Compton-scattered photons.

From these relations we calculate the ratio $I_{\mathrm{BS}}^{\text {total }} / I_{\mathrm{C}}^{\text {total }}$. In order to determine the relative contribution of BS in the region of the Compton profile (i.e. $-7 p_{0}$ to $+7 p_{0}$ ) we have used (1) to (4). The spectral distributions of BS from individual electron shells have been summed with proper weight for each value of $w$ through the photoelectric cross-section to yield the total atom contribution. The area of BS in the region of energies from 44 to $53 \mathrm{keV}$ corresponding to -7 to $+7 p_{0}$, relative to the total, is obtained from this. By combining these two steps we have computed the relative BS intensity in the region of Compton profiles $\left(-7\right.$ to $\left.+7 p_{0}\right)$ for each case. In another approach, we have repeated this procedure using the cross-section values from the self-consistent screened calculation of Lee et al. [8], which includes all the above corrections and is expected to yield similar results.

\section{Results and Discussion}

Using the procedure described in the last section, we have calculated the relative BS contribution for Mo, Ta and W. However, as a representative case we present detailed results for $\mathrm{W}$ only. The photo-ionisation cross-sections for W were taken from Rakavy and Ron [12]. As stated earlier, these values are modified for the Elwert and the form factor corrections using (2) 


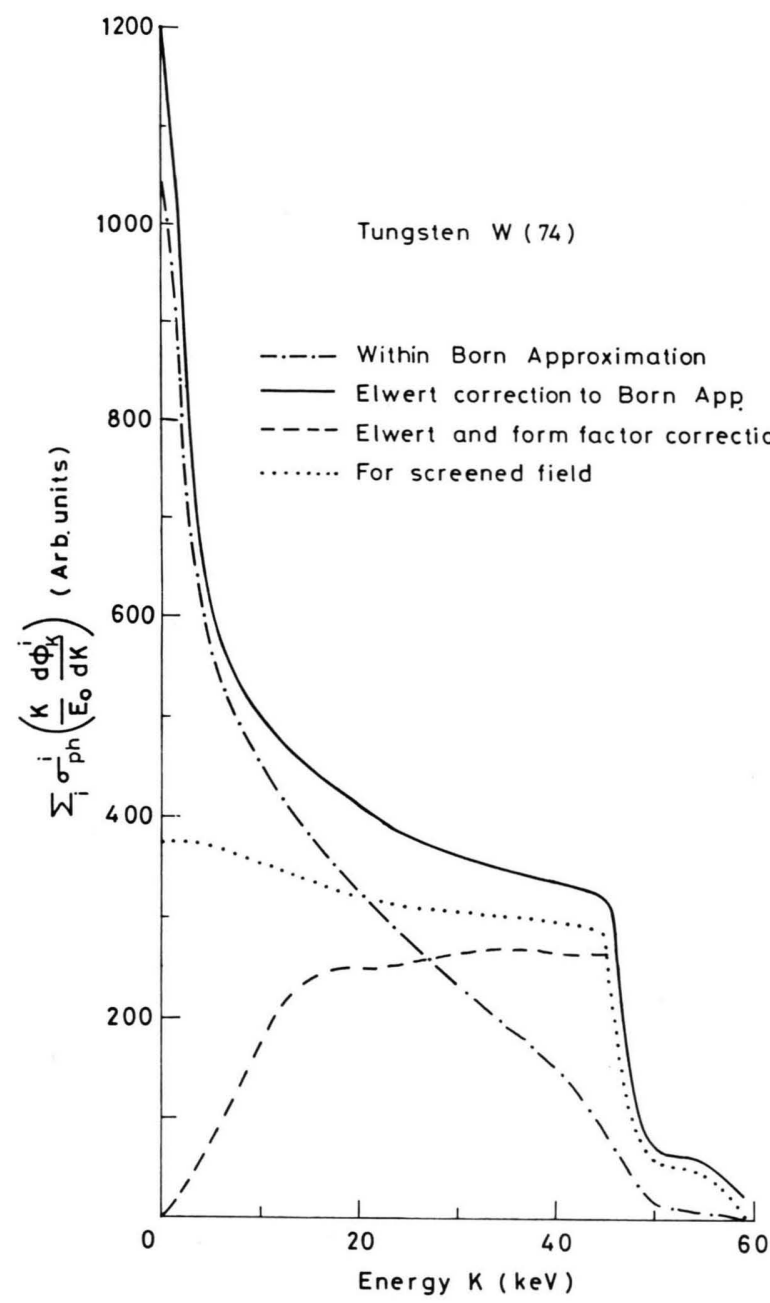

Fig. 1. Theoretical spectral distribution of bremsstrahlung calculated for decelerating electrons liberated in a photoelectric process resulting from $59.54 \mathrm{keV}$ gamma-rays.

Table 1. Relative intensities of bremsstrahlung (BS) and Compton scattered radiation.

\begin{tabular}{|c|c|c|c|c|}
\hline \multirow[t]{2}{*}{ Target } & \multirow{2}{*}{$\begin{array}{l}\text { Primary } \\
\text { energy }\end{array}$} & \multirow[t]{2}{*}{$I_{\mathrm{BS}}^{\text {total }} / I_{\mathrm{C}}^{\text {total }}$} & \multicolumn{2}{|c|}{$I_{\mathrm{BS}}^{-7 p_{0} \ldots+7 p_{0} * / I_{\mathrm{C}}^{\text {total }}}$} \\
\hline & & & $\begin{array}{l}\text { Born } \\
\text { approx. }\end{array}$ & $\begin{array}{l}\text { After } \\
\text { corrections }\end{array}$ \\
\hline Mo & $59.54 \mathrm{keV}$ & $3.27 \cdot 10^{-2}$ & 0.00024 & $0.0005^{1}$ \\
\hline $\mathrm{Ta}$ & $59.54 \mathrm{keV}$ & $5.99 \cdot 10^{-2}$ & 0.0014 & $\begin{array}{l}0.0041^{1} \\
0.0054^{2}\end{array}$ \\
\hline W & $59.54 \mathrm{keV}$ & $6.8 \cdot 10^{-2}$ & 0.0016 & $\begin{array}{l}0.0047^{1} \\
0.0069^{2} \\
0.0049^{3}\end{array}$ \\
\hline
\end{tabular}

1 After Elwert correction.

2 After Elwert and form factor correction.

3 For screened field.

* $p_{0}=1$ a.u. of momentum $=1.99289 \cdot 10^{-24} \mathrm{~kg} \mathrm{~m} / \mathrm{s}$. and (3). The form factor values have been taken from [10].

In Fig. 1 we plot the spectral distribution for different combinations of corrections, viz. the Born-approximation without further correction (BA), Elwert correction to the Born approximation (EBA) and Elwert and form factor (EBF). Also given here is the result from the screened field (SF) values [8]. In the first two cases we have taken the minimum value of $w$ as $0.1 \mathrm{keV}$, since the cross-section becomes infinite at zero photon energy. Figure 1 clearly depicts that these BS spectra overlap with the Compton-profile region (44 keV to $53 \mathrm{keV})$. Further, it can be seen from this figure that the EBA correction increases the BS contribution in the high-photon-energy range (i.e. from $20 \mathrm{keV}$ to $59 \mathrm{keV}$ ). Furthermore, the correction owing to the form factor produces an abrupt decrease in the low-photon-energy region $(15 \mathrm{keV})$, and hence the value of the cross-section for $0.1 \mathrm{keV}$ is nearly zero. The SF values [8] give an almost flat contribution from $w=0$ to $w=44 \mathrm{keV}$; after that it decreases abruptly and becomes zero at the tip $\left(w=T_{0}\right)$. This figure shows that the EBA, EBF and SF values are nearly equal after $20 \mathrm{keV}$. The abrupt decrease around $44 \mathrm{keV}$ arises owing to the fact that the L-electron contribution vanishes beyond this region and only outer electrons contribute to BS.

Since the BS intensity is proportional to the crosssection, the ratio of the areas under the curve in the energy region of the Compton profile and the total curve will be equal to the ratio of bremsstrahlung intensity given by $I_{\mathrm{BS}}^{-7 p_{0} \ldots+7 p_{0}} / I_{\mathrm{BS}}^{\text {total }}$. This has been determined from the above plot, and for the EBA and EBF curves the intensity ratio comes out to be 0.069 and 0.102 , respectively.

The intensity ratio $I_{\mathrm{BS}}^{\text {total }} / I_{\mathrm{C}}^{\text {total }}$ as calculated using (5) and (6) turns out to be 0.068 for $\mathrm{W}$. Therefore the values for $I_{\mathrm{BS}}^{-7} p_{0} \ldots+7 p_{0} / I_{\mathrm{C}}^{\text {total }}$ are 0.0047 and 0.0069 for EBA and EBF, respectively. For the screened-field approximation this value comes out to 0.0049 . These results for $\mathrm{W}$ along with those for the other two metals, viz. Mo and Ta, are summarised in Table 1.

Before we conclude this section, it is worthwhile to point out that Alexandropoulos et al. [5] have measured the spectrum of radiation when a gamma ray beam of $59.54 \mathrm{keV}$ was incident on a thick target of W. They have also calculated the BS spectrum using Kramer's formula for a thin target,

$$
B(\lambda) \mathrm{d} \lambda=1.8 \cdot 10^{-14} \Phi Z \cdot\left(\frac{\lambda}{\lambda_{0}}-1\right) \frac{\mathrm{d} \lambda}{\lambda^{2}},
$$


where $\Phi$ is the energy flux $(\mathrm{keV} / \mathrm{s})$ of the incident beam of energy $w_{0}=h c / \lambda_{0}$.

However, as is clear from Fig. 2 of [5], their theoretical curve agrees only up to $30 \mathrm{keV}$ with the experimental curve. The present results are not very different from these, although some differences do remain. Reliable values for the total bremsstrahlung contribution and photoionisation cross-section are therefore essential before any quantitative correction for BS can be made in Compton scattering experiments.

As far as Compton recoil electrons are regarded, the BS produced by the Compton electrons is limited to energies below $11 \mathrm{keV}$ in the present case, because the maximum energy transferred is about $11 \mathrm{keV}$ and its total contribution is much smaller than that produced by photoelectrons. Further, in the range $-7 p_{0}$ to $+7 p_{0}$, i.e. $44-53 \mathrm{keV}$, the contribution to BS by these electrons will be almost nil. However, if high-energy radiation is used then this contribution might also become important enough to be included in the calculations.

[1] B. Williams (ed.), Compton scattering, McGraw-Hill, London 1977.

[2] M. J. Cooper, Rep. Prog. Phys. 48, 415 (1985) and references therein.

[3] See for example, R. Benedek, R. Prasad, S. Manninen, B. K. Sharma, A. Bansil, and P. E. Mijnarends, Phys. Rev. B 32, 7650 (1985).

[4] J. Verma and M. A. Eswaran, Phys. Rev. 127, 1197 (1962).

[5] N. E. Alexandropoulos, T. Chatzigeorgiou, G. Evangelakis, M. J. Cooper and S. Manninen, Nucl. Instrum. Method A 271, 543 (1988) and references therein.

[6] U. Mittal, B. K. Sharma, F. M. Mohammad, and B. L. Ahuja, Phys. Rev. B 38, 12208 (1988).

[7] B. K. Sharma, U. Mittal, B. L. Ahuja, S. Perkkiö, S. Manninen, and T. Paakkari, in: Positron Annihilation

\section{Conclusion}

The BS spectrum overlaps the Compton-profile region, and its contribution is around $0.7 \%$ of the total Compton intensity for Ta and W. It can be minimised by selecting such a scattering angle that BS and scattered-beam spectra do not overlap. Otherwise we should apply a correction to the Compton profile for the BS contribution along with other corrections for high-precision Compton scattering data of the $5 \mathrm{~d}$ metals when using $59.54 \mathrm{keV}$ radiation.

\section{Acknowledgements}

This work is partially supported by the Department of Atomic Energy, India, via grant No. 37/11/89-G. Two of us (U.M. and R.K.K.) wish to thank the Director, College Education and the UGC, New Delhi, for the award of teacher-research-fellowships. One of us (B.L.A.) is grateful to DST, New Delhi, for a research project under Young Scientists Scheme.

and Compton scattering (B. K. Sharma, P. C. Jain, and R. M. Singru, eds.), Omega Scientific Publ., New Delhi 1990 , p. 261 , also p. 266.

[8] C. M. Lee, L. Kissel, R. H. Pratt, and H. K. Tseng, Phys. Rev. A 13, 1714 (1976).

[9] W. Heitler, The Quantum Theory of Radiation, Univ. Press, Oxford 1954. - H. W. Koch and J. W. Motz, Rev. Mod. Phys. 31, 920 (1959).

[10] J. H. Hubbel, W. J. Veigele, E. A. Biggs, R. T. Brown, D. T. Cromer, and R. J. Howerton, J. Phys. Chem. Ref. Data 4, 471 (1973).

[11] R. D. Evans, The Atomic Nucleus, Tata McGraw-Hill, New Delhi 1979.

[12] G. Rakavy and A. Ron, Phys. Rev. 159, 50 (1969). 